\title{
A scheme for amplification and discrimination of photons
}

\author{
A R Usha Devi ${ }^{1,2}, \mathbf{R}$ Prabhu $^{3,1}$ and A K Rajagopal ${ }^{4,2}$ \\ ${ }^{1}$ Department of Physics, Bangalore University, Bangalore-560 056, India \\ ${ }^{2}$ Inspire Institute Inc., McLean, VA 22101, USA \\ ${ }^{3}$ Department of Physics, Kuvempu University, Shankaraghatta, Shimoga-577 451, \\ India \\ ${ }^{4}$ Center for Quantum Studies, George Mason University, Fairfax, VA 22030, USA \\ E-mail: arutth@rediffmail.com
}

\begin{abstract}
A scheme for exploring photon number amplification and discrimination is presented based on the interaction of a large number of two-level atoms with a single mode radiation field. The fact that the total number of photons and atoms in the excited states is a constant under time evolution in Dicke model is exploited to rearrange the atom-photon numbers. Three significant predictions emerge from our study: Threshold time for initial exposure to photons, time of perception (time of maximum detection probability), and discrimination of first few photon states.

PACS numbers: 42.50.Ct, 03.65.Ta.
\end{abstract}

Submitted to: J. Phys. B: At. Mol. Phys. 


\section{Introduction}

A scheme for investigating amplification and discrimination of input photons is presented based on the interaction of a large number of identical two-level atoms and low intensity single mode radiation. This analysis provides a deeper understanding of the cumulative response of millions of atoms to few input photons within the basic model [1] of atomfield interaction.

Historically, collective behavior of $N$ two level atoms with single mode radiation, investigated by Dicke [1] in 1954, has led to vast array of interesting physical phenomena in quantum optics and recently, in artificial condensed matter systems [2]. The present investigation is an entirely novel application of the Dicke model. The fact that the sum of the number of photons and the number of atoms in the excited states is a constant under time evolution has a natural consequence of rearranging the atom-photon numbers resulting in photon amplification as well as discrimination of small number of photons. A projective measurement on temporally evolving combined multiatom-radiation system is shown to lead to both these features.

\section{Physical Model}

The Hamiltonian characterizing the interaction of $N$ two level atoms with a single mode radiation is given by $(\hbar=1)$

$$
H=\omega\left(a^{\dagger} a\right)+\omega_{0} S_{z}+\frac{g}{\sqrt{N}}\left(S_{+} a+S_{-} a^{\dagger}\right)
$$

where $g$ denotes the atom-photon coupling parameter; $\omega_{0}$, the atomic splitting and $\omega$ the filed frequency; $a^{\dagger}(a)$ are creation (annihilation) operators of the field satisfying the bosonic commutation relations $\left[a, a^{\dagger}\right]=1$. The collective (pseudo) spin operators of the two-state atoms

$$
S_{ \pm}=S_{x} \pm i S_{y}=\frac{1}{2} \sum_{\alpha=1}^{N} \sigma_{\alpha \pm}, S_{z}=\frac{1}{2} \sum_{\alpha=1}^{N} \sigma_{\alpha z}
$$

obey the commutation relations

$$
\left[S_{+}, S_{-}\right]=2 S_{z}, \quad\left[S_{z}, S_{ \pm}\right]= \pm S_{ \pm} .
$$

It is well-known [3] that the excitation operator

$$
N_{\mathrm{ex}}=a^{\dagger} a+S_{z}+\frac{N}{2}
$$

remains constant as the system evolves under the Hamiltonian (11). The degenerate atom-photon states $\left|S=\frac{N}{2}, M=n_{e}-\frac{N}{2}\right\rangle \otimes|n\rangle\left(-\frac{N}{2} \leq M \leq \frac{N}{2}\right.$, or equivalently $n_{e}=0,1,2, \ldots, N$, and $\left.n=0,1,2, \ldots\right)$, with a fixed eigenvalue $\ddagger n+n_{e}$ of (4) span the space of the Hamiltonian. The symmetric atomic Dicke states $\left|S=\frac{N}{2}, M=n_{e}-\frac{N}{2}\right\rangle$

$\ddagger$ The Fock states $|n\rangle, n=0,1,2, \ldots$ denote eigenstates of the photon number operator $a^{\dagger} a$ with eigenvalue $n$ and the Dicke states $\left|S=\frac{N}{2}, M\right\rangle,-\frac{N}{2} \leq M \leq \frac{N}{2}$ are joint eigenstates of $S^{2}=S_{x}^{2}+S_{y}^{2}+S_{z}^{2}$ and $S_{z}$ with eigenvalues $\frac{N}{2}\left(\frac{N}{2}+1\right), M$ respectively. 
(except for $n_{e}=0$ and $N$ ) are well-known for their entanglement properties [4, 5, 6]. These are essential in the ensuing discussion \&. The collective ground state of the atomic system is denoted by $|0\rangle=\left|\frac{N}{2},-\frac{N}{2}\right\rangle$ throughout this sequel.

A given initial state of the combined atom-radiation system, $\rho_{A R}(0)$ evolves to

$$
\rho_{A R}(t)=e^{-i H t} \rho_{A R}(0) e^{i H t} .
$$

Consequently, a projection operator $\Pi_{A 0} \otimes I_{R}$, with $\Pi_{A 0}=|0\rangle\langle 0|$, and $I_{R}$ the unit operator in the radiation space, gives us the conditional density matrix of the collective atom-photon system ((where all the atoms are projected to their collective ground state)), subjected to the constraint (4). This projective measurement has the implication that maximum number of photons are emitted consequently. The collective atomic ground state serves as a detection device and the projection operation at subsequent suitable intervals of time corresponds to efficient measurement of the radiation state containing maximum number of photons allowed. It may be observed that the constant of motion (4) leads to rearrangement of the number of atoms in the excited state with the number of photons, leading to photon amplification. This is evident if $n_{e}>n$ number of atoms are initially in excited state with $n$ input photons, in which case $\left\langle N_{\mathrm{ex}}\right\rangle=n+n_{e}$ and a subesquent measurement projects the atoms to their collective ground state leading to $\left\langle a^{\dagger} a\right\rangle(t)=n+n_{e}$. More generally, $\left\langle a^{\dagger} a\right\rangle(t)=\left\langle N_{\mathrm{ex}}\right\rangle-\left\langle S_{z}\right\rangle(t)-\frac{N}{2}$. A judicious choice of the initial state and the time of projection measurement is the basis of photon amplification in this model. When a pure collective low lying excited state of atoms are considered, the method outlined above results in photon number discrimination as well.

\section{Photon amplification with different initial states}

\subsection{Pure atom-photon state $\left|n_{e} ; n\right\rangle$}

Let us consider an initial atom-photon state

$$
\left|S=\frac{N}{2}, M=n_{e}-\frac{N}{2}\right\rangle \otimes|n\rangle \equiv\left|n_{e} ; n\right\rangle,
$$

with $n_{e}<<N$, and the number of atoms $N$ sufficiently large so that the HolsteinPrimakoff mapping [7]

$$
S_{+}=b^{\dagger} \sqrt{N-b^{\dagger} b}, S_{-}=\sqrt{N-b^{\dagger} b} b, S_{z}=b^{\dagger} b-\frac{N}{2}
$$

in terms of bosonic operators $b, b^{\dagger}$ satisfying

$$
\left[b, b^{\dagger}\right]=1,
$$

reduces the Hamiltonian (11) into a two mode bosonic interaction structure [2] (up to order $\mathcal{O}(1 / N))$ :

$$
H \sim \omega N_{\mathrm{ex}}-\omega_{0} \frac{N}{2}+g\left(b^{\dagger} a+b a^{\dagger}\right) .
$$

$\S$ Dicke states with a specific permutation symmetry are chosen here because of the greatest simplicity offered by them in their theoretical analysis and also due to their current experimental relevance [4, 5]. 
Temporal evolution in this approximation thus assumes the form,

$$
U(\tau)=e^{-i H t}=e^{-i \tau\left(\frac{\omega}{g} N_{\mathrm{ex}}-\frac{\omega_{0}}{2 g} N\right)} e^{-i \tau\left(b^{\dagger} a+b a^{\dagger}\right)} .
$$

(Here we have denoted $g t=\tau$.) It may be noted that $U(\tau)$ acts as a passive unitary beam-splitter on the two mode bosonic Fock states $\left|n_{e} ; n\right\rangle$. Thus, initial states of the form (66) get confined within the space spanned by the $\left(n_{e}+n+1\right)$ basis states $\left|n_{e}^{\prime} ; n^{\prime}\right\rangle$, with $n_{e}^{\prime}, n^{\prime}=0,1,2 \ldots$ such that $n_{e}^{\prime}+n^{\prime}=n_{e}+n$ under time evolution. It is interesting to note that at scaled time $\tau=\pi / 2$ the unitary operator $U(\tau)$ swaps $[8]$ the atom-photon numbers i.e., $\left|n_{e} ; n\right\rangle \rightarrow\left|n ; n_{e}\right\rangle$ with unit probability.

In general, we have,

$$
\begin{gathered}
U(\tau)\left|n_{e} ; n\right\rangle=e^{-i \tau\left[\frac{\left(n+n_{e}\right) \omega}{g}+\frac{N \omega_{0}}{2 g}\right]} \sum_{n_{e}^{\prime}, n^{\prime}}\left|n_{e}^{\prime}, n^{\prime}\right\rangle\left\langle n_{e}^{\prime}, n^{\prime}\left|e^{-i \tau\left(b^{\dagger} a+b a^{\dagger}\right)}\right| n_{e}, n\right\rangle \\
=e^{-i \tau\left[\frac{\left(n+n_{e}\right) \omega}{g}+\frac{N \omega_{0}}{2 g}\right]} \sum_{n_{e}^{\prime}, n^{\prime}}\left|n_{e}^{\prime}, n^{\prime}\right\rangle d_{\frac{n_{e}^{\prime}-n^{\prime}}{2}, \frac{n_{e}-n}{2}}^{\frac{n_{e}+n}{2}}(2 \tau) \\
\times e^{-i \pi\left[\left(n_{e}^{\prime}-n^{\prime}\right)-\left(n_{e}-n\right)\right] / 4} \delta_{n_{e}^{\prime}+n^{\prime}, n_{e}+n},
\end{gathered}
$$

where 9 ]

$$
\begin{aligned}
d_{m^{\prime}, m}^{j}(2 \tau)=\sum_{k} & \frac{\sqrt{(j+m) !(j-m) !\left(j+m^{\prime}\right) !\left(j-m^{\prime}\right) !}}{(j+m+k) ! k !\left(j-k-m^{\prime}\right) !\left(k-m+m^{\prime}\right) !}(-1)^{k-m+m^{\prime}} \\
& \times(\cos \tau)^{2 j-2 k+m-m^{\prime}}(\sin \tau)^{2 k-m+m^{\prime}}=d_{m, m^{\prime}}^{j}(-2 \tau)
\end{aligned}
$$

with the sum over $k$ taken such that none of the arguments of the factorials in the denominator are negative.

A measurement $\Pi_{A 0} \otimes I_{R}$ at an instant $t$ projects all the atoms to ground state:

$$
\begin{aligned}
\Pi_{A 0} \otimes I_{R} U(\tau)\left|n_{e} ; n\right\rangle & =\sum_{n^{\prime}}\left|0, n^{\prime}\right\rangle d_{-\frac{\left(n+n_{e}\right)}{2}, \frac{n_{e}-n}{2}}^{\frac{n_{e}+n}{2}}(2 \tau) \delta_{n^{\prime}, n_{e}+n} \\
& \times e^{i \pi\left[\left(n_{e}-n+n^{\prime}\right)\right] / 4} e^{-i \tau\left[\frac{\left(n+n_{e}\right) \omega}{g}+\frac{N \omega_{0}}{2 g}\right]} \\
= & e^{-i t\left(n \omega+\frac{N}{2} \omega_{0}\right)} e^{-i n_{e}\left(\omega t-\frac{\pi}{2}\right)}(-1)^{n_{e}} \sqrt{\mathcal{P}\left(n_{e}, n, \tau\right)}\left|0 ; n+n_{e}\right\rangle .
\end{aligned}
$$

Thus, the probability of finding the atoms in ground state, which in turn corresponds to that of photon amplification $n \rightarrow n+n_{e}$, is given by

$$
\mathcal{P}\left(n_{e}, n, \tau\right)=\left(\begin{array}{c}
n+n_{e} \\
n_{e}
\end{array}\right) \cos ^{2 n}(\tau) \sin ^{2 n_{e}}(\tau)
$$

In Figs. 1 (a), (b), (c), we have displayed the probability of maximum photon emission for three choices of $n_{e}=1,10$, and 25, each with different values for the number of photons $n=0,1,5,10$, as a function of scaled time $\tau$. For $n=0$ i.e., dark photon input state, the probability has a single peak $\llbracket$ in the range $0 \leq \tau \leq \pi$ with maximum value $\mathcal{P}\left(n_{e}, 0, \tau=\pi / 2\right)=1$, which corresponds to swapping of atom-photon numbers $\left|n_{e} ; 0\right\rangle \rightarrow\left|0 ; n_{e}\right\rangle$. However, when $n \neq 0$ the projective measurement $\Pi_{A 0} \otimes I_{R}$ leads to $\left|n_{e} ; n\right\rangle \rightarrow\left|0 ; n+n_{e}\right\rangle$ corresponding to maximum photon emission; this is not a

$\|$ The probability $\mathcal{P}\left(n_{e}, n, \tau\right)$ exhibits repetitive structure with a period $\pi$, as is evident from (14). 
(a)

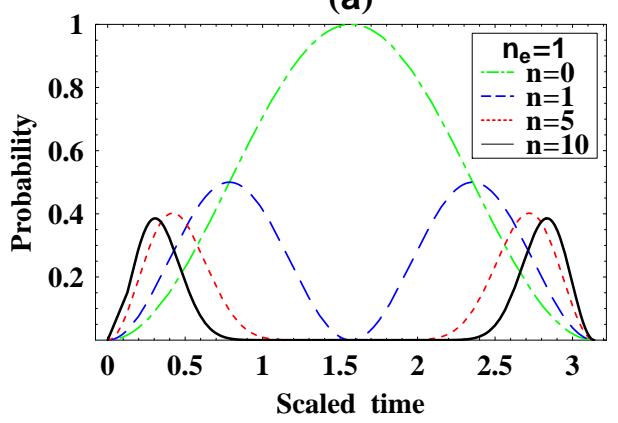

(b)

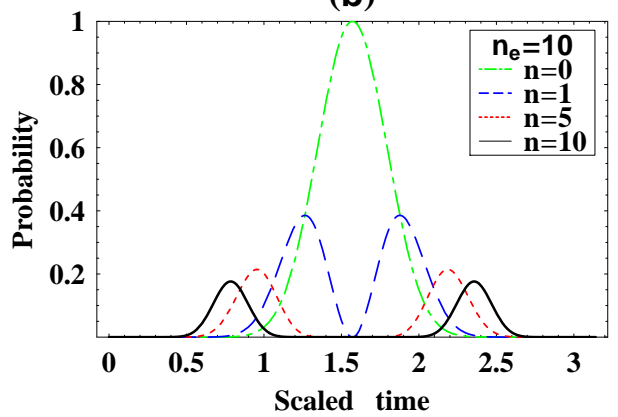

(c)

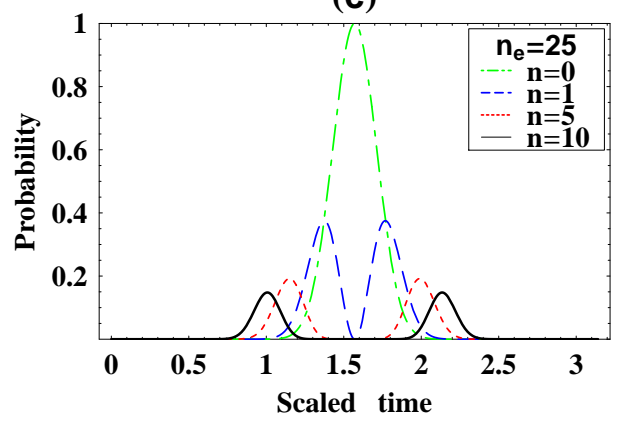

Figure 1. Probability $\mathcal{P}\left(n_{e}, n, \tau\right)$ of finding the atoms in ground state as a function of scaled time $\tau$ for different values of initial photon numbers $n$. Maxima in probabilities occur at scaled times $\tau_{n}=\arccos \sqrt{\frac{n}{n+n_{e}}}$.

complete swap action and occurs with probability $0 \leq \mathcal{P}\left(n_{e}, n \neq 0, \tau\right)<1$. Clearly, the probability $\mathcal{P}\left(n_{e}, n \neq 0, \tau\right)$ vanishes at scaled time $\tau=\pi / 2$, in order to give way to complete swap action $\left|n_{e} ; n\right\rangle \rightarrow\left|n_{e} ; n\right\rangle$. The probability profile for maximum photon emission $n \rightarrow n+n_{e}$ therefore reveals two peaks around $\tau=\pi / 2$ for $n \neq 0$. The maxima of probabilities corresponding to different input photon numbers are well separated and they appear periodically at $\tau_{n}=\arccos \sqrt{\frac{n}{n+n_{e}}}$, allowing for photon number discrimination.

We define the time of perception $\tau_{p}\left(n_{e}, n\right)$, for a given $n_{e}$ and $n$, as the time at which the probability $\mathcal{P}\left(n_{e}, n, \tau\right)$ attains its maximum. This determines the efficient detection of the photons. An examination of these figures reveal: (i) for a given $n_{e}$, the time of perception $\tau_{p}\left(n, n_{e}\right)$ reduces as more and more photons are detected - with lesser and lesser efficiency. Moreover the widths in the probabilities $\mathcal{P}\left(n_{e}, n, \tau\right)$ reduce correspondingly. (ii) As $n_{e}$ increases, there is a threshold time for the detection of photons, before which the probabilities are zero. In particular, for $n_{e}=1$, there is an instant response to photons (for all $n$ ) as seen in Fig. 1(a), whereas for higher values of $n_{e}$ there is a delay in such a response (See Fig. 1. (b), and (c)). (iii) For a given photon number $n$ the profile of probability as a function of time sharpens as $n_{e}$ increases; however maximum value of the probability drops with this, as is evident from Figs. 1. 


\subsection{Pure state of atoms with low intensity coherent radiation}

The above discussion was confined to pure photon number states. We now show that an enhanced photon amplification behavior is realized, when initially a low intensity coherent state of radiation

$$
|\alpha\rangle=e^{-|\alpha|^{2} / 2} \sum_{n=0}^{\infty} \frac{\alpha^{n}}{\sqrt{n !}}|n\rangle,|\alpha|^{2}<1
$$

is considered as input. The probability of finding the atoms in ground state is obtained by following the procedures given in (11) and (13):

$$
\mathcal{P}\left(n_{e}, \alpha, \tau\right)=e^{-|\alpha|^{2}} \sum_{n=0}^{\infty} \mathcal{P}\left(n_{e}, n, \tau\right) \frac{|\alpha|^{2 n}}{n !} .
$$
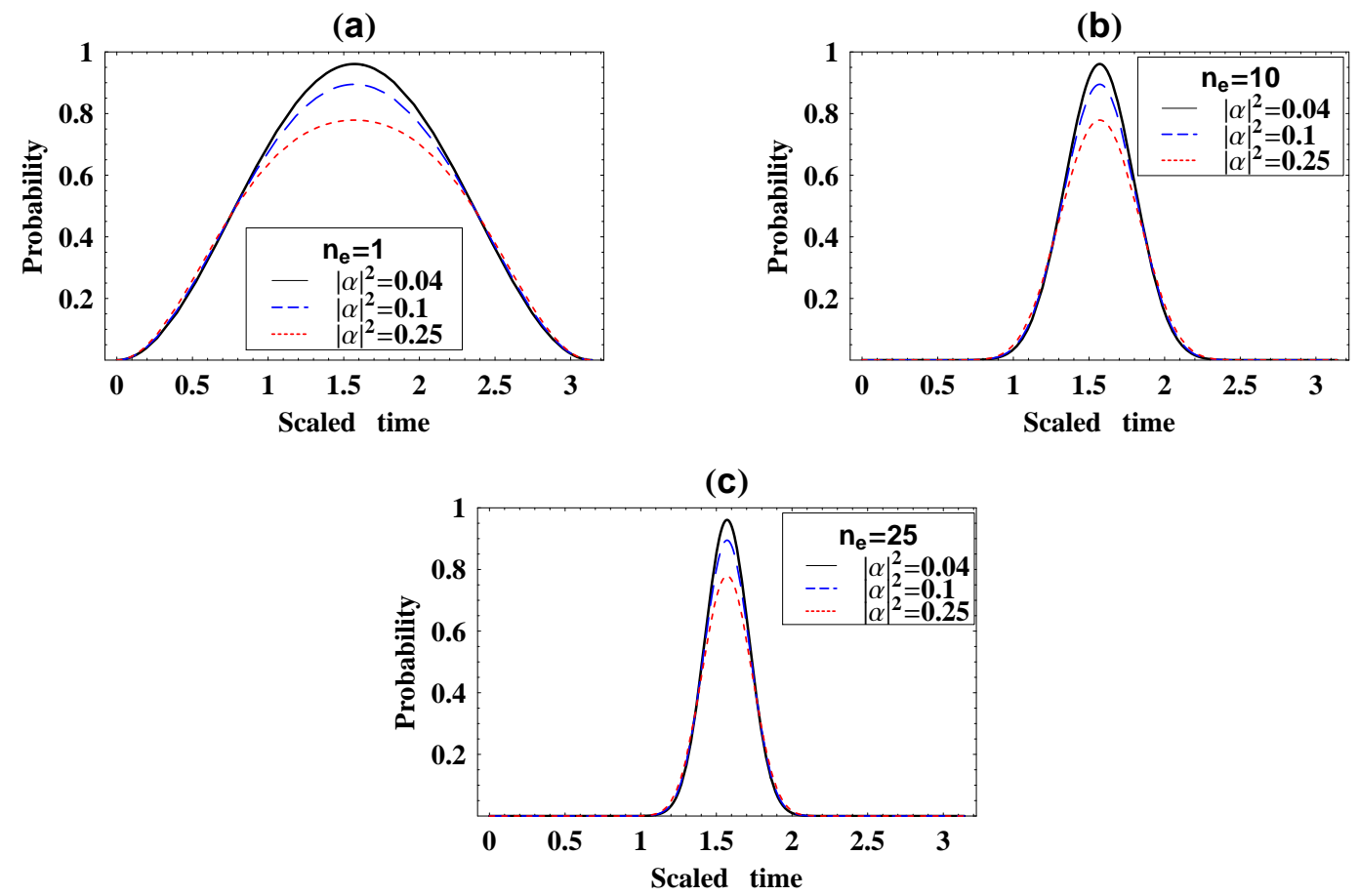

Figure 2. Probability $\mathcal{P}\left(n_{e}, \alpha, \tau\right)$ of finding the atoms in ground state as a function of scaled time $\tau$ for different values of initial intensity $|\alpha|^{2}$ and for different choices of $n_{e}$, the initial number of atoms in excited state.

The probability profile (16) - with coherent radiation as input - is a series involving individual atom-photon Fock state probabilities $\mathcal{P}\left(n_{e}, n, \tau\right)$, dark photon probability $\mathcal{P}\left(n_{e}, 0, \tau\right)$ being the leading term for low value of intensity $|\alpha|^{2}$. Thus, the probability response to low intensity coherent light has a similar structure as that of dark photon probability $\mathcal{P}\left(n_{e}, 0, \tau\right)$ (see Fig. 1) with small contribution from higher order terms $|\alpha|^{2 n}, n=1,2, \ldots$. It may be seen from Fig. 2 that the probability $\mathcal{P}\left(n_{e}, \alpha, \tau\right)$ is found to be nearly zero in the begining and raises to a maximum at $\tau=\pi / 2$ (peak value being $\left.\mathcal{P}\left(n_{e}, \alpha, \pi / 2\right) \sim e^{-|\alpha|^{2}}\right)$. An increase in the initial intensity of coherent radiation has the effect of reducing the probability of finding the atoms in their ground state. 
(a)

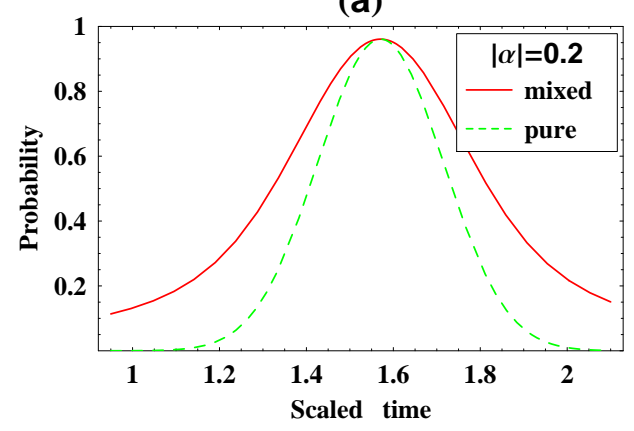

(b)

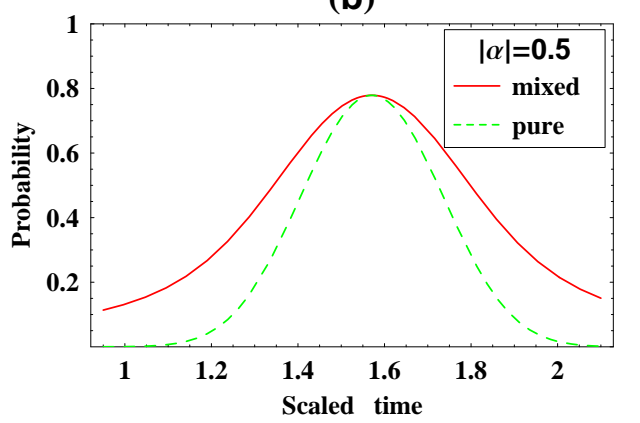

Figure 3. Probability of finding the atoms in ground state under time evolution governed by the Hamiltonian (9), when initial atomic state is chosen as (i) a mixed state $\frac{1}{n_{e}+1} \sum_{m=0}^{n_{e}}|m\rangle\langle m|$, (ii) pure state $\left|n_{e}\right\rangle$, and the radiation in a coherent state, $|\alpha\rangle$, as a function of scaled time $\tau$, for two different values of inital intensity of radiation and $n_{e}=25$.

Amplification of the intensity of radiation, $\frac{I(t)}{I(t=0)}=\frac{\left\langle a^{\dagger} a\right\rangle(t)}{|\alpha|^{2}}$, (after performing projective measurement $\Pi_{A 0}$ on the temporally evolving state $\left.U(\tau)\left|n_{e} ; \alpha\right\rangle\right)$ approaches the value $\frac{n_{e}}{|\alpha|^{2}}$ as $\tau \rightarrow \frac{\pi}{2}$ i.e., when the probability of finding the atoms in ground state is maximum.

\subsection{Mixed state of atoms with low intensity coherent radiation}

So far, we have considered pure atomic states. It is of interest to investigate how the effects found above may get modified when mixed atomic states are employed. For simplicity, we choose here a chaotic mixture $\left(n_{e}+1\right)$ of low lying collective excited states of atoms,

$$
\rho_{\text {atom }}=\frac{1}{n_{e}+1} \sum_{m=0}^{n_{e}}|m\rangle\langle m|, \quad n_{e}<<N,
$$

along with low intensity coherent radiation. The probability of finding the atoms in ground state under time evolution in this model is readily found to be,

$$
\mathcal{P}\left(\rho_{\text {atom }}, \alpha, \tau\right)=\frac{e^{-|\alpha|^{2}}}{n_{e}+1} \sum_{m=0}^{n_{e}} \sum_{n=0}^{\infty} \frac{|\alpha|^{2 n}}{n !} \mathcal{P}(m, n, \tau) .
$$

The probability $\mathcal{P}\left(\rho_{\text {atom }}, \alpha, \tau\right)$ as well as $\mathcal{P}\left(n_{e}, \alpha, \tau\right)$ (see Eq. (16)) associated with a pure atomic state $\left|n_{e}\right\rangle$ are plotted, as a function of scaled time $\tau$, in Fig. 3 (a) and (b) for two different choices of the initial intensity of radiation for a fixed $n_{e}=25$. We observe that while the maximum probability remains the same for a given initial intensity of radiation $|\alpha|^{2}$, the mixed states $\rho_{\text {atom }}$ result in a wider spread around the maximum value (at $\tau=\pi / 2$ ) compared to their pure state counterparts. Also, the probability $\mathcal{P}\left(\rho_{\text {atom }}, \alpha, \tau\right)$ builds above the background value $\frac{1}{n_{e}+1}$. Moreover, as the intensity of the radiation $|\alpha|^{2}$ increases, the maximum probability drops down. It may be noted that the photon amplification factor $\frac{I(t)}{I(0)}$ approaches the value $\frac{n_{e}}{2|\alpha|^{2}}$ as $\tau \rightarrow \pi / 2$ for mixed atomic states $\rho_{\text {atom }}$ in contrast to its corresponding value $\frac{n_{e}}{|\alpha|^{2}}$ in the case of pure states $\left|n_{e}\right\rangle$. 


\section{Summary}

We have presented a scheme for photon amplification and discrimination based on the Dicke model interaction between single mode radiation and $N$-atom system. This involves Holstein-Primakopf large $N$ approximation [7, where the number $n_{e}$ of atoms in excited states is assumed to be much smaller than the total number $N$ of atoms. Further, this approximation enforces that the number $n$ of input photons is small, compared to $N$. This leads to a beam splitter feature for time evolution under the interaction Hamiltonian (9), thus ensuring entanglement between the atoms in collective excited states and the photons [10]. Emission of maximum number of photons corresponds to a completely uncorrelated atom-radiation system, with all the atoms in the ground state and this is acheived via a projective measurement at a suitable interval of time. Concepts like threshold time and time of perception for exposure to low intensity light emerge as characteristic features of our investigation. Discrimination of small number of photons is also realized in this scheme. This provides motivation for a quantum mechanical analysis of the collective response of millions of rods in the eye, which act as nearly perfect photon detectors, initiating the associated process of vision during night [11, 12, 13].

\section{Acknowledgement}

We thank the Referees for their insightful comments, which improved the presentation of our work in this revised version.

\section{References}

[1] Dicke R H 1954 Phys. Rev. 9399

[2] Brandes T 2005 Phys. Rep. 408315

[3] Tavis M and Cummings F W 1967 Phys. Rev. 170 379; 1969 Phys. Rev. 188692

[4] Thiel C, von Zanthier J, Bastin T, Solano E and Agarwal G S 2007 Phys. Rev. Lett. 99193602

[5] Bastin T, Thiel C, von Zanthier J, Lamata L, Solano E and Agarwal G S 2007 arXiv:0710.3720

[6] Usha Devi A R, Prabhu R and Rajagopal A K 2007 Phys. Rev. A 76012322

[7] Holstein T and Primakoff H 1949 Phys. Rev. 581098

[8] Xiaoguang Wang, 2001 J. Phys. A: Math. Gen. 349577

[9] Sakurai J J 1999 Modern Quanum Mechanics (New York: Addison-Wesley) p 223

[10] Wang Xiang-bin 2002 Phys. Rev. A 66024303

[11] Kandel E R, Schwartz J H and Jassel T M 2000 Principles of Neural Science (New York: McGrawHill)

[12] Ramakrishna Chakravarthi, Rajagopal A K and Usha Devi A R 2008 To appear in the Proceedings of the Indo-US Workshop on Science and Technology at the Nano-Bio Interface (February 19-22, 2008, Bhubaneswar, India)

[13] Rieke F and Baylor D A 1998 Rev. Mod. Phys. 701027 